\title{
An Adaptive Threshold Algorithm for Joint Sparse Recovery
}

\author{
N. F. Dong, J. X. Wang \\ School of Electronic and Optical Engineering \\ Nanjing University of Science and Technology \\ Nanjing, China
}

\begin{abstract}
This paper presents a new greedy algorithm for joint sparse recovery, called adaptive threshold simultaneous orthogonal matching pursuit (AT-SOMP). In this algorithm, an adaptive threshold is designed based on subspace decomposition to stop the iterative process, during which the support of target signal is identified iteratively just like the simultaneous orthogonal matching pursuit (S-OMP) algorithm. As the adaptive threshold can change with the noise power adaptively, the proposed algorithm is applied to the situation that both the sparsity and SNR of the target signal are unknown. Experiments validate that in this situation the proposed algorithm has a better recovery performance than those methods with a fixed threshold.
\end{abstract}

Keywords-compressed sensing; joint sparse recovery; adaptive threshold; simultaneous orthogonal matching pursuit

\section{INTRODUCTION}

Compressed sensing (CS) [1] has received increasing interests over the past few years. According to CS theory, a sparse signal $\mathbf{x}$ can be reconstructed approximately from the measurement vector $\mathbf{y}=\mathbf{A x}$ obtained through the sensing matrix $\mathbf{A}$. A natural extension of this recover problem is the joint sparse recovery problem, also known as the multiple measurement vector (MMV) problem where a single matrix $\mathbf{A}$ is utilized to obtain measurements of multiple signals: $\mathbf{y}_{k}=\mathbf{A} \mathbf{x}_{k}$ for $k=1, \cdots, N$. The joint sparse recovery problem aims to identify a common support shared by unknown sparse vectors $\left\{\mathbf{x}_{k}\right\}_{k=1}^{N}$ from the multiple measurements vectors $\left\{\mathbf{y}_{k}\right\}_{k=1}^{N}$. Once the support is determined, the recovery of the unknown sparse vectors reduces to a standard overdetermined linear inverse problem.

Algorithms have been developed for the joint sparse recovery problem. Convex optimization formulations with mixed norm [2-4] extend the corresponding single measurement vector (SMV) solution, such as basis pursuit (BP) [5] and LASSO [6] to the MMV case. They work correctly and provide theoretical performance guarantees. However, they often suffer from heavy computational complexity when solving massive recovery problems. On the other hand, greedy algorithms such as orthogonal matching pursuit (OMP) [7] and compressive sampling matching pursuit (CoSaMP) [8] were extended to the MMV case [9-11]. Greedy algorithms iteratively identify the support of the target signal until a halting condition is met. They can solve large-scale reconstruction problems more efficiently, but there is no optimal way to stop the algorithms when the sparsity is unknown. One common approach is to halt when a relative residue improvement between two consecutive iterations is smaller than a certain threshold. However, the certain threshold is hard to design in the situation that the signal to noise ratio (SNR) is unknown, and an inappropriate threshold may cause poor reconstruction performance of the recovery algorithm.

In this paper, a new greedy algorithm, called adaptive threshold simultaneous orthogonal matching pursuit (ATSOMP), is proposed for joint sparse recovery. The AT-SOMP algorithm combines simultaneous orthogonal matching pursuit (S-OMP) algorithm with an adaptive threshold designed based on subspace decomposition. It iteratively identifies the support of the target signal just like S-OMP and halts when a residue improvement between two consecutive iterations is smaller than the adaptive threshold. As the adaptive threshold changes with the noise power adaptively, the AT-SOMP algorithm can works well with unknown sparsity in the situation that SNR is unknown. Experiments validate that the AT-SOMP algorithm outperforms those methods with a fixed threshold in the case that the sparsity and SNR of the target signal are unknown. The rest of this paper is organized as follows. Section 2 provides an introduction to the signal model and the S-OMP algorithm for the MMV case. In Section 3, we present the design of the adaptive threshold. Finally, the simulation results are shown in Section 4, and the conclusion is provided in Section 5.

\section{REVIEW}

\section{A. Signal Model}

Symbol $\mathbb{K}$ denotes a scalar field, which is either the real field $\mathbb{R}$ or the complex field $\mathbb{C}$. Let $\left\{\mathbf{x}_{k}\right\}_{k=1}^{N} \subset \mathbb{K}^{n}$ be jointly $s$-sparse (i.e., the union of the supports of $\mathbf{x}_{k}$ for $k=1, \cdots, N$ has at most ${ }_{s}$ elements). Then, $\mathbf{X}=\left[\mathbf{x}_{1}, \cdots, \mathbf{x}_{N}\right] \in \mathbb{K}^{n \times N}$ has no more than $s$ nonzero rows. The support of $\mathbf{X}$ denotes the union of the supports of the columns of $\mathbf{X}$, or equivalently, the set of indices of the nonzero rows.

Utilizing an $m \times n$ sensing matrix $\mathbf{A}$, we obtains the $m \times N$ observation matrix $\mathbf{Y}$ :

$$
\mathbf{Y}=\mathbf{A} \mathbf{X}+\mathbf{W}
$$


where $\mathbf{W}$ is an $m \times N$ noise matrix. Let $J_{0}$ denote the support of $\mathbf{X}$ and let $\mathbf{X}^{J_{0}}$ denote the submatrix with the nonzero rows of $\mathbf{X}$. Then the matrix $\mathbf{Y}$ can be expressed as

$$
\mathbf{Y}=\mathbf{A}_{J_{0}} \mathbf{X}^{J_{0}}+\mathbf{W}
$$

where $\mathbf{A}_{J_{0}}$ is a submatrix containing the columns of $\mathbf{A}$ indexed by $J_{0}$. As the Gaussian assumption is one of the most widely used in signal processing, we assume that all the elements in the $i$ th row of the matrix $\mathbf{X}^{J_{0}}$ are independent Gaussian variables of variance $\sigma_{i}^{2}$. Then eqn. (2) can be written in compact form:

$$
\mathbf{Y}=\mathbf{A}_{J_{0}} \cdot \boldsymbol{\Sigma}^{1 / 2} \cdot \mathbf{U}+\mathbf{W}
$$

where $\mathbf{U}$ is an $s \times N$ random matrix with independent standard Gaussian entries and $\boldsymbol{\Sigma}$ is an $s \times s$ diagonal matrix whose diagonal entries $\sigma_{i}^{2}$ are positive real numbers.

\section{B. S-OMP Algorithm}

S-OMP algorithm, an extension of OMP algorithm, is an iterative algorithm for the MMV case. In this setting, one should combine the correlation of the atom $\mathbf{A}_{r}$ (the $r$ th column of the matrix $\mathbf{A}$ ) with the multiple vectors to get a single correlation criterion for the selection of the most correlated atoms. For any $1 \leq p \leq \infty$, one can consider the $p$-correlation

$$
\left\|\mathbf{A}_{r}^{H} \mathbf{Y}\right\|_{p}=\left(\sum_{k=1}^{N}\left|\left\langle\mathbf{A}_{r}, \mathbf{Y}_{k}\right\rangle\right|^{p}\right)^{1 / p}
$$

As input, the S-OMP algorithm requires three pieces of information: the $m \times n$ sensing matrix $\mathbf{A}$, the $m \times N$ observation matrix $\mathbf{Y}$, and a halting criterion. The algorithm is initialized with the residual $\mathbf{R}_{0}=\mathbf{Y}$ and the support set $J^{0}=\varphi$. At the $i$ th iteration, S-OMP performs four major steps:

1) Identify the index ${ }_{r_{i}}$ of the atom ${ }_{\mathbf{A}_{r_{i}}}$ which maximizes the $p$-correlation $\left\|\mathbf{A}_{r_{i}}^{H} \mathbf{R}_{i-1}\right\|_{p}=\max _{1 \leq r \leq n}\left\|\mathbf{A}_{r}^{H} \mathbf{R}_{i-1}\right\|_{p}$ with the residual $\mathbf{R}_{i-1} \cdot$

2) The newly identified index $r_{i}$ is united with the support set $J^{i-1}$ to get the new set $J^{i}=J^{i-1} \cup r_{i}$.

3) Solve a least-squares problem $\hat{\mathbf{X}}^{J^{i}}=\mathbf{A}_{J^{i}}^{\dagger} \mathbf{Y}$ to approximate the target signal on the merged support $J^{i}$.

4) Calculate the new residual $\mathbf{R}_{i}=\mathbf{Y}-\mathbf{A}_{J^{i}} \hat{\mathbf{X}}^{J^{i}}$.

These steps are repeated until the halting criterion is triggered. In the experiments in Section 4, the S-OMP algorithm adopts the existing common halting condition:

$$
\frac{\left\|\mathbf{R}_{i-1}\right\|_{F}-\left\|\mathbf{R}_{i}\right\|_{F}}{\left\|\mathbf{A}_{J_{i}} \hat{\mathbf{X}}^{J^{i}}\right\|_{F}} \leq \varepsilon
$$

where $\varepsilon$ is a certain threshold.

\section{ADAPTIVE THRESHOLD}

In this section, an adaptive threshold is designed based on subspace decomposition to create a halting criterion for our AT-OMP algorithm. We assume that the columns of the noise matrix $\mathbf{W}$ are i.i.d. random vectors with white spectrum (i.e., $\left.\mathbb{E}\left(\frac{\mathbf{W W}^{H}}{N}\right)=\sigma_{w}^{2} \mathbf{I}_{m}\right)$ and the number of snapshots $N$ is large but finite (specifically, $N$ satisfies $N \geq m$ ). It is also assumed that $m \geq 2 s$, which is required by most blind recovery methods.

Multiplying the observation matrix $\mathbf{Y}=\mathbf{A}_{J_{0}} \mathbf{X}^{J_{0}}+\mathbf{W}$ by its conjugate transpose, we have

$$
\Gamma_{\mathbf{Y}}=\mathbf{Y} \mathbf{Y}^{H} \text {. }
$$

Let $\left\{\lambda_{i}\right\}_{i=1}^{m}$ denote the eigenvalues of the matrix $\boldsymbol{\Gamma}_{\mathbf{Y}}$ and $\lambda_{1} \geq \lambda_{2} \geq \cdots \geq \lambda_{m}$. Then $\left\{\lambda_{i}\right\}_{i=1}^{s}$ represent energies of signal subspaces and the other eigenvalues represent energies of noise subspaces. Although the sparsity $s$ is unknown, we know that the eigenvalue $\lambda_{d+1}$ is the energy of a noise subspace, where $d=\lfloor m / 2\rfloor$ is the largest integer not greater than $m / 2$.

According to the iterative process described in the previous section, an index $r_{i}$ is identified and a residual $\mathbf{R}_{i}=\mathbf{Y}-\mathbf{A}_{J_{i}} \hat{\mathbf{X}}^{J_{i}}$ is updated at the $i$ th iteration. When $i \leq s$, one signal subspace of $\mathbf{X}^{J_{0}}$ will be recovered and the residue improvement $\left\|\mathbf{R}_{i-1}\right\|_{F}^{2}-\left\|\mathbf{R}_{i}\right\|_{F}^{2}$ will be great compared to the eigenvalue $\lambda_{d+1}$. After $s$ iterations, the support $J_{0}$ will be completely recovered. Then, if the iterative process continues, a noise subspace will be recovered and $\left\|\mathbf{R}_{i-1}\right\|_{F}^{2}-\left\|\mathbf{R}_{i}\right\|_{F}^{2}$ will be close to $\lambda_{d+1}$ (even less than $\lambda_{d+1}$ ). Therefore, in the situation that the sparsity $s$ is unknown, the eigenvalue $\lambda_{d+1}$ can be employed to halt the iterative process.

From the above mentioned, we propose a new halting condition: the iteration stops when

$$
\left\|\mathbf{R}_{i-1}\right\|_{F}^{2}-\left\|\mathbf{R}_{i}\right\|_{F}^{2}<\tau \lambda_{d+1}
$$

where $\tau$ is a rational number greater than 1. Empirical results suggest that the choice $\tau=2.5$ is often reasonable. As the adaptive threshold $\tau \lambda_{d+1}$ can change with the noise power adaptively, the proposed halting condition is better than the previous halting criterions with fixed thresholds especially in the situation that SNR is unknown. 


\section{EXPERIMENTS}

In this section, we compare the performance of the proposed AT-SOMP algorithm with S-OMP and sparsity adaptive matching pursuit (SAMP) [12] experimentally. The sensing matrix is generated as randomly selected $m$ rows of the $n \times n$ DFT matrix. The observation matrix $\mathbf{Y}=\mathbf{A X}+\mathbf{W}$ follows the model in eqn. (3) and is corrupted by additive i.i.d. circular complex Gaussian noise $\mathbf{W}$. Set $m=20$ and $n=64$. The algorithms are tested on random row $s$-sparse matrix $\mathbf{X}$ with uniformly random support $J_{0}$. The power $\sigma_{1}^{2}$ of the minimum signal in $\mathbf{X}^{J_{0}}$ is set to 1 and the other power $\left\{\sigma_{i}^{2}\right\}_{i=2}^{s}$ is chosen uniformly random in $[0, \mathrm{SNR}-10]^{\mathrm{dB}}$ compared with $\sigma_{1}^{2}$. The performance is assessed by the rate of successful support recovery.

The major interest of the first experiment is how many snapshots $N$ are needed in AT-SOMP algorithm to reconstruct different sparse signals. The SNR is set to $20 \mathrm{~dB}$. For each pair of $s$ and $N, 10000$ random matrix $\mathbf{X}$ are employed to calculate the rate of successful support recovery. We show the results in fig. 1. As expected, the performance improves with increasing $N$. And it is shown that $N=30$ is large enough for our AT-SOMP algorithm in the case that $m=20$.

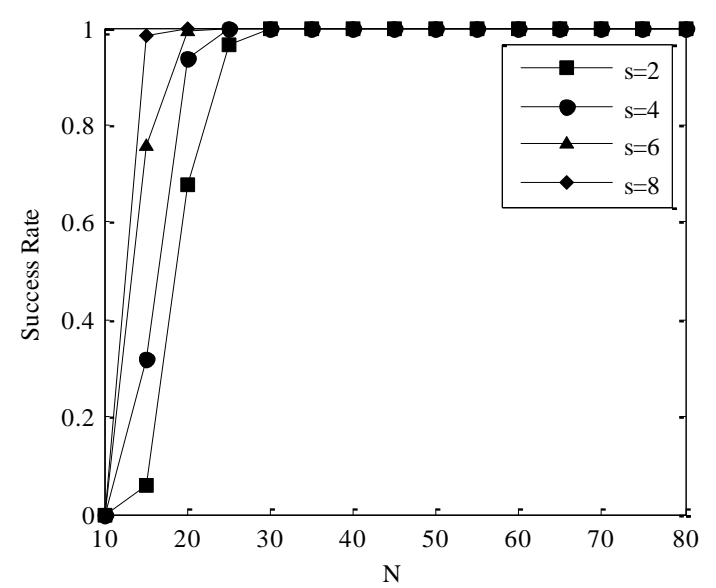

\section{FIGURE I. SUCCESS RATE VERSUS SNAPSHOT NUMBER $N$ FOR DIFFERENT SPARSE SIGNALS WITH SNR=20DB}

In the second experiment, we compare the performance of AT-SOMP with S-OMP and SAMP under different SNR levels. The threshold $\varepsilon_{\varepsilon}$ of S-OMP and SAMP is set to two levels: 0.02 and 0.002. The other parameters are set to $s=6$ and $N=64$. For each SNR, the success rate is calculated by attempting recovery of 10000 random row $s$-sparse matrix $\mathbf{X}$. As shown in fig. 2, a fixed threshold of S-OMP and SAMP just works for several SNR levels and our AT-OMP algorithm works well for all the SNR levels.

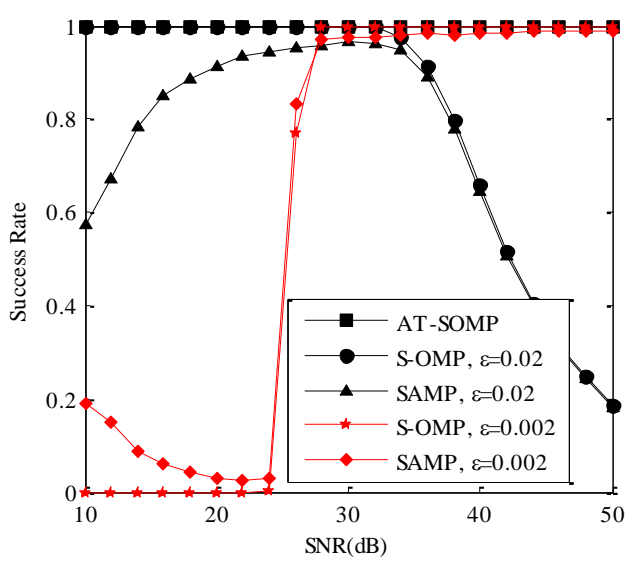

FIGURE II. SUCCESS RATE VERSUS DIFFERENT SNRS WITH $s=6$

$$
\text { AND } N=128
$$

\section{CONCLUSIONS}

A new greedy algorithm called AT-SOMP for joint sparse recovery is proposed in this paper. It iteratively identify the support of the target signal just like S-OMP and halts when a residue improvement between two consecutive iterations is smaller than the adaptive threshold designed based on subspace decomposition. Experiment results demonstrate the AT-SOMP algorithm is a powerful algorithm compared to those methods with a fixed threshold in the case that the sparsity and SNR of the target signal are unknown.

\section{REFERENCES}

[1] Donoho, D., Compressed sensing. IEEE Transactions on Information Theory, 52(4), pp. 1289-1306, 2006.

[2] Tropp, J., Algorithms for simultaneous sparse approximation. Part II: Convex relaxation. Signal Processing, 86(3), pp. 589-602, 2006.

[3] Chen, J. \& Huo, X., Theoretical results on sparse representations of multiple-measurement vectors. IEEE Transactions on Signal Processing, 54(12), pp. 4634-4643, 2006.

[4] Obozinski, G., Wainwright, M. \& Jordan, M., Support union recovery in high-dimensional multivariate regression. The Annals of Statistics, 39(1), pp. 1-47, 2011.

[5] Chen, S., Donoho, D. \& Saunders, M., Atomic decomposition by basis pursuit. SIAM Review, 43(1), pp. 129-159, 2001.

[6] Tibshirani, R., Regression shrinkage and selection via the lasso: a retrospective. Journal of the Royal Statistical Society: Series B, 73(3), pp. 267-288, 1996.

[7] Pati, Y., Rezaiifar, R. \& Krishnaprasad, P., Orthogonal matching pursuit: Recursive function approximation with applications to wavelet decomposition. Proc. of the $27^{\text {th }}$ Asilomar Conf. on Signals, Systems and Computers, pp. 40-44, 1993.

[8] Needell, D. \& Tropp, J., CoSaMP: Iterative signal recovery from incomplete and inaccurate samples. Applied and Computational Harmonic Analysis, 26(3), pp. 301-321, 2009.

[9] Tropp, J., Gilbert, A. \& Strauss, M., Algorithms for simultaneous sparse approximation. Part I: Greedy pursuit. Signal Processing, 86(3), pp. 572-588, 2006.

[10] Gribonval, R., Rauhut, H., Schnass, K. \& Vandergheynst, P., Atoms of all channels, unite! Average case analysis of multi-channel sparse recovery using greedy algorithms. Journal of Fourier Analysis and Applications, 14(5), pp. 655-687, 2008.

[11] Blanchard, J., Cermak, M., Hanle, D. \& Jing, Y., Greedy algorithms for joint sparse recovery. IEEE Transactions on Signal Processing, 62(7), pp.1694-1704, 2014.

[12] Do, T., Gan, L., Nguyen, N. \& Tran, T., Sparsity adaptive matching pursuit algorithm for practical compressed sensing. Poc. of the $42^{\text {nd }}$ Asilomar Conf. on Signals, Systems and Computers, pp. 581-587, 2008. 